This item was submitted to Loughborough's Research Repository by the author.

Items in Figshare are protected by copyright, with all rights reserved, unless otherwise indicated.

\title{
LQG control of a high redundancy actuator
}

PLEASE CITE THE PUBLISHED VERSION

PUBLISHER

(C) IEEE

VERSION

VoR (Version of Record)

LICENCE

CC BY-NC-ND 4.0

\section{REPOSITORY RECORD}

Du, Xinli, Roger Dixon, Roger M. Goodall, and Argyrios C. Zolotas. 2019. "LQG Control of a High Redundancy Actuator". figshare. https://hdl.handle.net/2134/4297. 
This item was submitted to Loughborough's Institutional Repository (https://dspace.lboro.ac.uk/) by the author and is made available under the following Creative Commons Licence conditions.

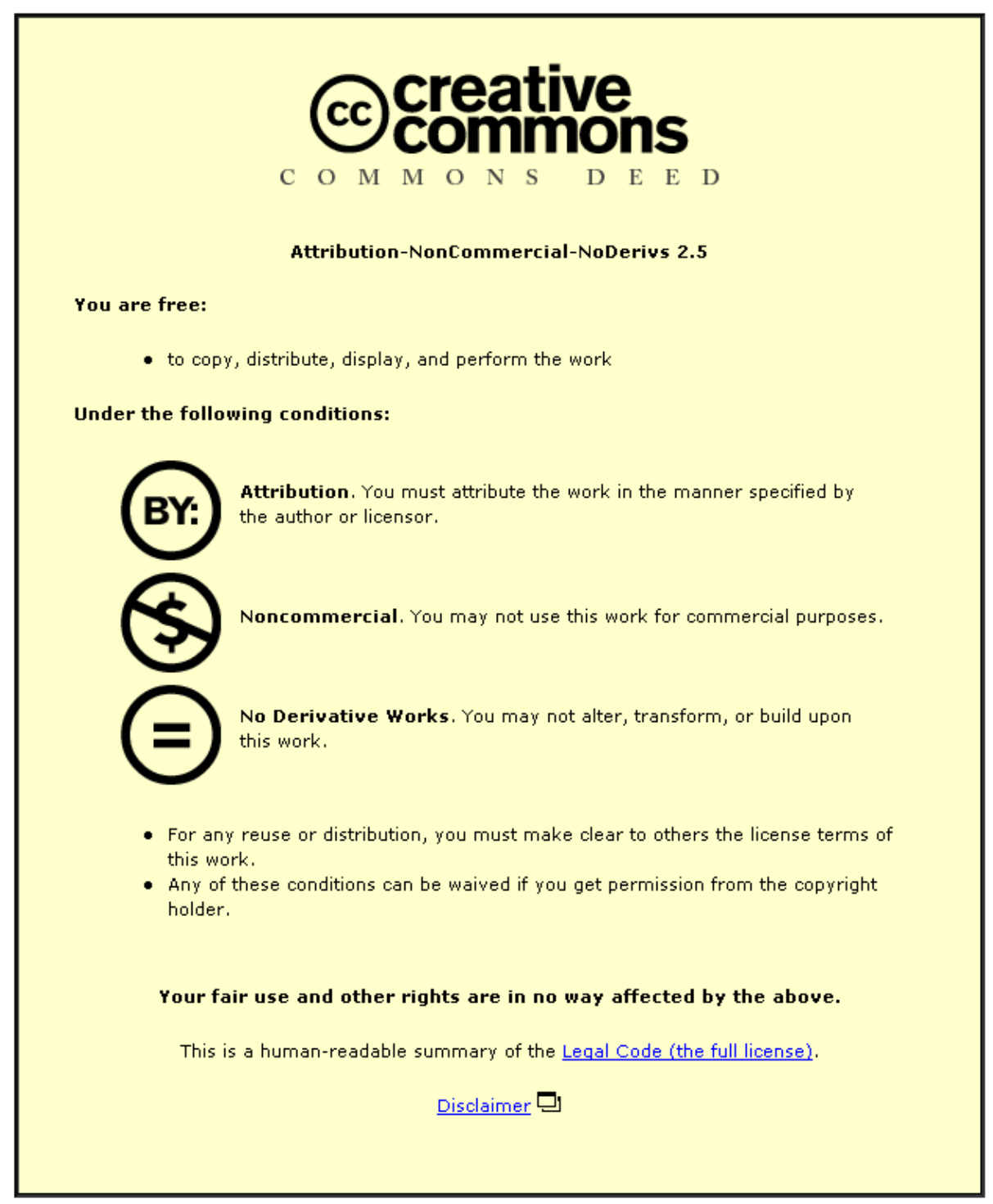

For the full text of this licence, please go to: http://creativecommons.org/licenses/by-nc-nd/2.5/ 


\title{
LQG Control of a High Redundancy Actuator
}

\author{
X. Du, R. Dixon, R. M. Goodall, and A. C. Zolotas \\ Loughborough University, Loughborough, Leicestershire, LE11 3TU, U.K. \\ Tel. +44 (0)1509 227015, Email: x.du@lboro.ac.uk
}

\begin{abstract}
A high redundancy actuator, comprising a relatively large number of actuation elements, is being developed for safety critical applications. Some classical control results have previously been reported and this paper will focus on evaluation of the LQG control design. Three different design approaches will be presented and compared under different types of typical faults in the sub-actuation elements. Overall a LQG design using a physically motivated reduced order model appears to be the best approach.
\end{abstract}

Copyright (C 2007 LOUGHBOROUGH UNIVERSITY

Index Terms - fault tolerance, high redundancy, LQG control

\section{INTRODUCTION}

High levels of availability and reliability are important objectives for the design of most modern engineering systems, especially in safety critical applications. Hence fault tolerant systems, which have the capability of tolerating component malfunctions whilst still maintaining desirable and robust performance and stability properties [1], are the solution to such problems. Such tolerance should not only be built into hardware/mechanisms but also in the controller design.

Practical examples of fault tolerant systems can be found in aerospace systems, e.g. Airbus fly-by-wire system [2] and Boeing 737 trailing edge flap drive system [3]. Here, low levels of functional redundancy in sensors and actuators (e.g. triplex and quadruplex) and even in the control computers can be used to provide the system with the capability of fault tolerance, thereby ensuring the safety and stability of the whole system.

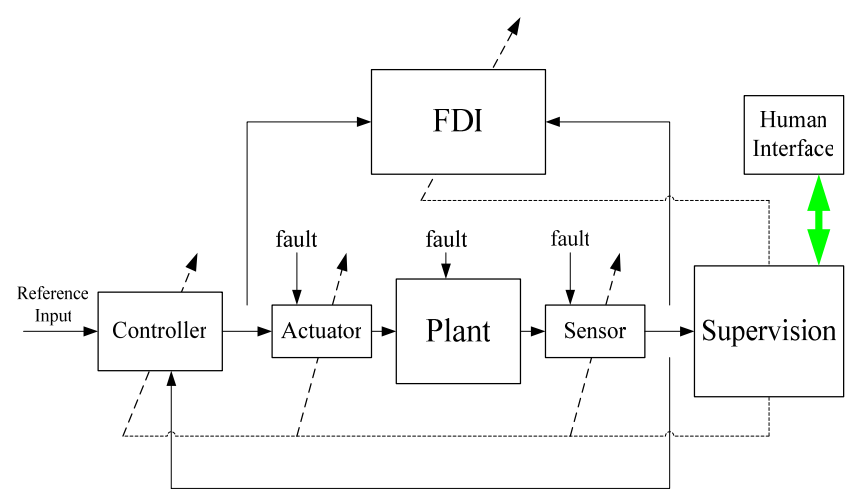

Fig. 1 Scheme of fault-tolerant control system with supervision subsystem [1]
A popular structure of fault tolerant system, as proposed in [1], includes a fault detection and isolation (FDI) unit and reconfiguration scheme which as shown in Fig 1. The solid lines represent signal flow, and the dashed lines represent adaptation. The supervision system will reconfigure the actuator and/or sensor sets, and adapt the controller, based on the fault information collected by the FDI unit together with the inputs and outputs of the system, to accommodate the fault effects. A possible disadvantage of such approaches is the possibility of faults occurring in the FDI unit and the supervision system. One might ask the question, "Who/what monitors the monitors?".

Another approach, called passive fault tolerance, uses a fixed robust controller that tolerates changes of the plant dynamics [2]. The method is called passive because fault tolerance is obtained without changing parameters or structures of the controller. However, the robust control theory is limited to a relatively small range of changes in the plant behaviour caused by faults.

This paper proposes an alternative route to fault tolerant actuation. The high redundancy actuator (HRA) suggested comprises a relatively large number of actuation elements in a matrix-like structure, and is controlled in such a way that faults in individual actuation elements are inherently accommodated. A fault detection unit may still be required for monitoring, but it is no longer strictly necessary and no reconfiguration of the controller or hardware will be needed. Instead the actuation elements work together to complete the system's objective by using the redundancy which is inherent in the structure. The HRA, working with a fixed controller, extends the limit of robust control theory in fault tolerant applications by using a more complex mechanical configuration. If this method can be proven to work, the advantage would arise through removing the possibility of faults occurring within the FDI unit and supervision system.

Several possible configurations are shown in Fig 2. At this stage no attempt has been made to find the optimal structure for the actuation elements but to concentrate upon the most appropriate control approach although some discussions relating to the configurations are discussed in a related paper [5]. Using a relatively simple (2 by 2 ) structure, a classical controller has been designed and reported in previous papers with a view to discovering whether controlling such structures without reconfiguration is viable $[6,7]$. 


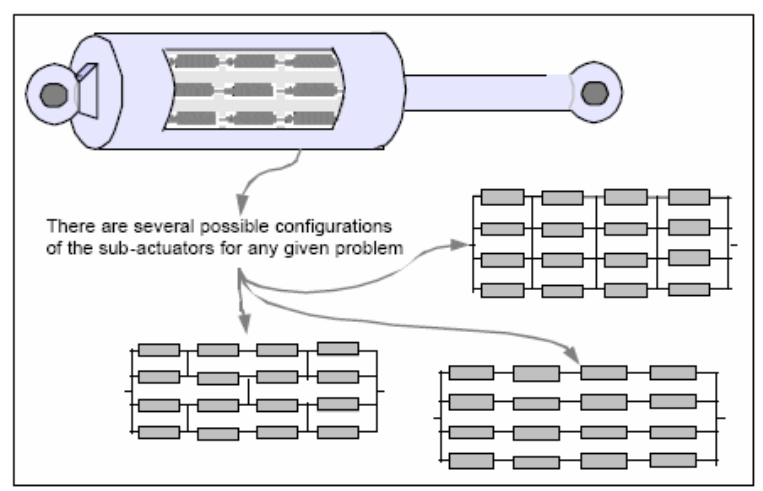

Fig. 2 Several possible configurations of the high redundancy actuator

This paper will focus on the LQG design. Hence, the paper is structured as follows: work on modelling is presented in section 2; the control design using a LQG approach is covered in section 3 ; then in section 4 a selection of simulation results is presented to give an indication of performance in the presence of faults alongside the fault-free case; the paper concludes in section 5, including comments on the future direction of this research.

\section{Modelling WorK}

\section{A. Individual Actuator Model}

Electromechanical actuators are chosen as the sub-elements. The simulation model built in Simulink/MATLAB can be found in $[6,7]$. It can be presented as a linear state space model in the well known form of $\dot{x}=A x+B u \quad y=C x$, where

$$
\begin{gathered}
A=\left[\begin{array}{ccccccc}
-\frac{R_{a m m}}{L_{a m m}} & -\frac{K_{e}}{L_{a m}} & 0 & 0 & 0 & 0 & 0 \\
\frac{K_{t}}{J_{m}} & -\frac{C_{m}}{J_{m}} & -\frac{n^{2} K_{m}}{J_{m}} & 0 & \frac{n K_{m}}{J_{m}} & 0 & 0 \\
0 & 1 & 0^{*} & 0 & 0 & 0 & 0 \\
0 & 0 & \frac{K_{m}{ }^{*} n}{M_{s}} & -\frac{C_{s}}{M} & -\frac{K_{s}+K_{m}}{M_{s}} & \frac{C_{s}}{M} & \frac{K_{s}}{M_{s}} \\
0 & 0 & 0 & 1 & 0 & 0 & 0 \\
0 & 0 & 0 & \frac{C_{s}}{M} & \frac{K_{s}}{M} & -\frac{C_{s}}{M} & -\frac{K_{s}}{M} \\
0 & 0 & 0 & 0 & 0 & 1 & 0
\end{array}\right], \\
B=\left[\begin{array}{ccccccc}
\frac{1}{L_{a r m}} & 0 & 0 & 0 & 0 & 0
\end{array}\right]^{T}, C=\left[\begin{array}{lllllll}
0 & 0 & 0 & 0 & 0 & 1 & 1
\end{array}\right] \\
x=\left[\begin{array}{llllllll}
\dot{i}_{a} & \dot{\theta}_{m} & \theta_{m} & \dot{x}_{m} & x_{m} & \dot{x}_{a c t} & x_{a c t}
\end{array}\right]^{T}, u=v_{a}, y=\left[\begin{array}{llll}
\dot{x}_{a c t} & x_{a c t}
\end{array}\right]^{T}
\end{gathered}
$$

TABLE I

DEFINITION OF PARAMETERS AND STATES

\begin{tabular}{|c|c|c|c|}
\hline$R_{\text {arm }}$ & motor resistance & $L_{\text {arm }}$ & inductance of the windings \\
\hline$K_{e}$ & voltage constant & $K_{t}$ & torque constant \\
\hline$J_{m}$ & motor inertia & $C_{m}$ & damping coefficient \\
\hline$K_{m}$ & motor stiffness & $n$ & screw pitch \\
\hline$K_{s}$ & screw stiffness & $C_{s}$ & Screw damping \\
\hline$M_{s}$ & screw mass & $M$ & load mass \\
\hline$i_{a}$ & armature current & $\theta_{m}$ & angular rotation of the motor shaft \\
\hline$x_{m}$ & screw position & $x_{a c t}$ & end-of-actuator position \\
\hline
\end{tabular}


Lockup: The actuator becomes locked into position due to mechanical interference within the mechanism. This fault is serious as it cannot be solved (by reconfiguration) at the signal level.

\section{LQG CONTROL}

Optimal control seeks to control the plant so as to get the best possible performance which is expressed in a mathematical expression (a cost function). It is based on state variable models of systems. Through mathematical methods (generally computer-assisted), a state variable feedback matrix will be designed to minimize the cost function to achieve the optimal performance. The Linear Quadratic Regulator (LQR) uses a simple quadratic cost function expressed as follows:

$$
J=\int_{t_{0}}^{t_{f}}\left[x^{T} Q x+u^{T} R u\right] d t .
$$

With modern computer-assisted control system design (CACSD) environments (such as MATLAB Control System Toolbox), the result from minimizing the cost function can be adjusted to obtain the required closed-loop performance by tuning weighting matrix $\mathrm{Q}$ and $\mathrm{R}$. Based on the LQR controller design method, the Linear Quadratic Gaussian (LQG) approach combines optimal state estimation (via a Kalman filter) with the LQR controller design. It helps to avoid disturbance and noise, but only based on accurate plant models and information about white noise disturbances. Here the controller design will be started with an individual actuator. Then, the same process will be applied to the two by two series-in-parallel structure. In both cases the aim is to control the load position in response to a command input (tracking control).

\section{A. Control of Individual Actuator}

Previous papers $[6,7]$ studied the individual and multiple actuators in frequency domain. An open-loop individual actuator Nichols chart is given in Fig 5 using position as output. A high gain is needed to achieve a better performance in frequency domain, which has been proved in the classical design. The system is fully controllable and observable which gives the possibility of LQG control design.

In the LQG control design, an extra state, which is the integral of position error, is added into the previous seven states individual actuator model to ensure tracking of the position command. The corresponding weight for the extra state in $\mathrm{Q}$, which is chosen as a diagonal matrix, is relatively large compared with the other seven weights, while the input weighting matrix $\mathrm{R}$ is chosen as single value because only one control input is considered here. Position and velocity measurements are chosen as the input to the state estimator. The simulation result with a $0.03 \mathrm{~m}$ step input is shown in the Fig 6.

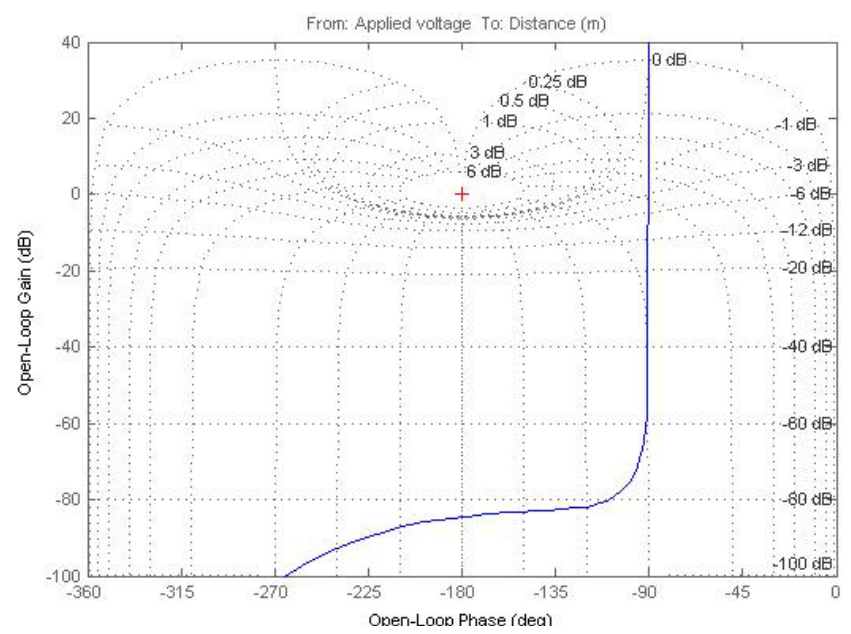

Fig. 5 Open-loop Nichols chart for individual actuator
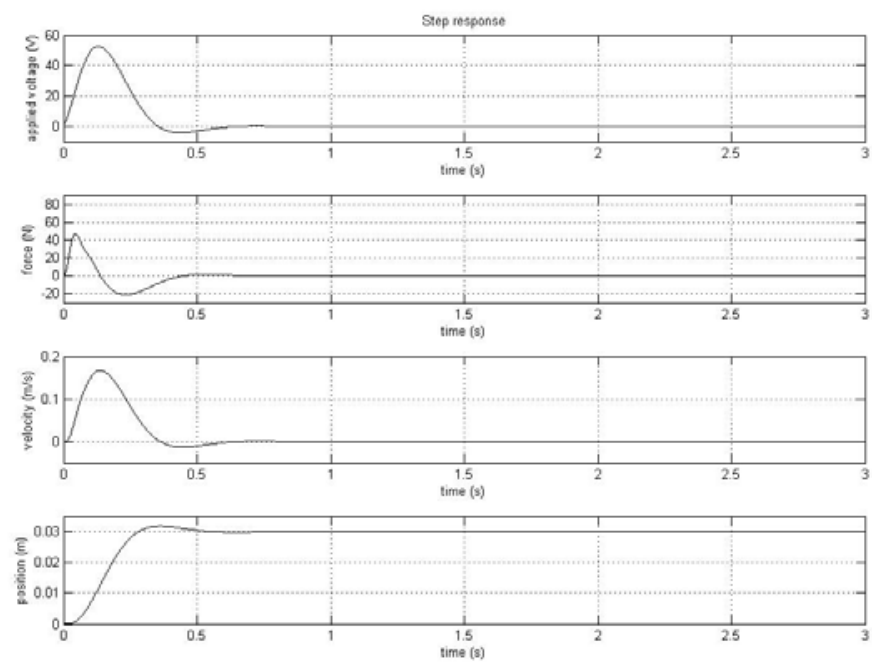

Fig. 6 Control result for individual actuator

\section{B. Control of Multiple Actuator}

The controller design is based on the series-in-parallel two by two structure which has been shown in Fig 4. In the simulation, the four sub-actuation elements are ideally the same so that the frequency performance for the SP is similar to the individual actuator. However, the repeated dynamics reduce the controllability and observability of the system which causes mathematical problems for the LQG control design. To obviate this problem, three different approaches will be presented. The first approach is to add some small variations into the parameters to avoid the repetition. The other two methods employ model reduction. The full order system includes 26 states (as introduced in section II) and the final two approaches attempt to reduce the order, thereby alleviating the problem. Of these, one uses a mathematical method called balanced realization truncation and the other uses a physical reduction (based on the physical equations).

1) Parameter Variation: Five percent variations of each parameter are introduced to each actuation element in different place. As introduced in the section II B, the HRA model includes four individual actuator models (also some connection items). The variation in actuation element helps to avoid the repeated dynamics of each sub element which 
would otherwise make calculation of the controller and observer gains difficult.

2) Balanced Realization Truncation: The central problem in model reduction is to find a low-order approximation given a high-order linear time-invariant stable model such that the infinity norm of the difference is small. The advantage is that a simpler controller can be found by reducing the number of states. The balanced realization truncation is based on the balanced realization of the model which evaluates the contributions to the response of each mode [9]. The state coordinate basis is selected as a diagonal matrix in descending order. The magnitudes of the diagonal entries reflect the contributions. Only the most effective states, which affect the input-output mostly, are kept so that similar performance still can be achieved. Again, it's relatively easy to find the balanced realization and the most effective states in the realization of the SP two by two HRA with the help of CACSD environments (MATLAB Control System Toolbox). In this case, eight states are kept in the balanced truncation model

3) Physical Reduction: As for the balanced realization, the purpose for physical reduction is to find a low-order model which has similar performance to the high-order model. The difference here is that the reduction is based on physical understanding rather than mathematical methods. In this approach, the series-in-parallel two by two actuator structure is seen as a bigger individual actuator with four times power input, but double speed and position outputs. This approach gives a state space model with only seven states, just as an individual actuator model. The simulation results show very similar performance between the full order and reduced order model in both frequency and time domain although difference can be found in high frequency which is not important in this case for the controller design.

Then, same progress as the individual actuator control design can be applied while different models are used in the state estimator (Kalman filter). Again, the integral of position error is added as an extra state into the model, with a relatively large corresponding weight in $\mathrm{Q}$ matrix, to ensure tracking of the position command.
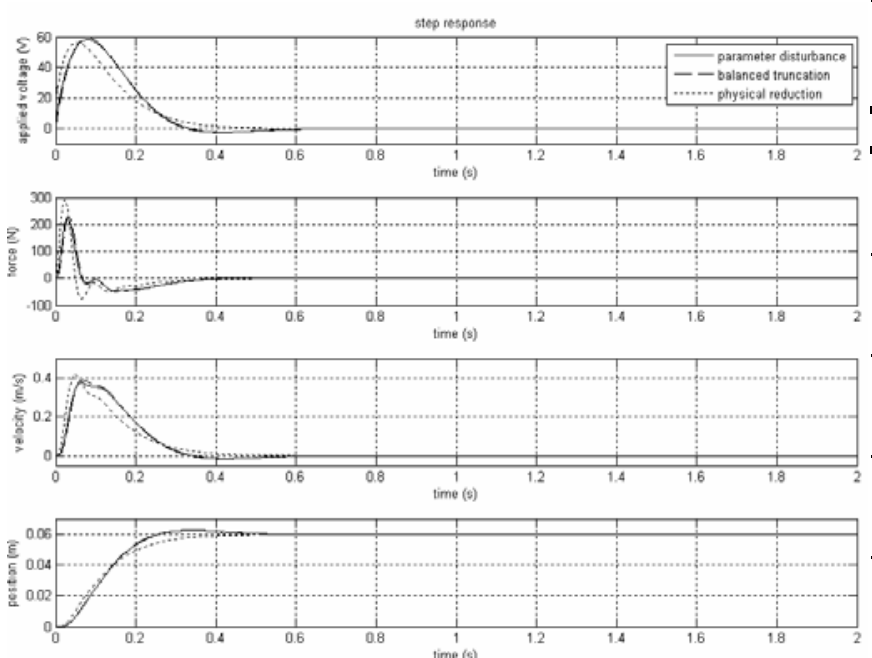

Fig. 7 Global control result for two by two structure
The simulation results with a $0.06 \mathrm{~m}$ step input using the three different approaches described above is given in Fig 7. All four sub-actuation elements are connected to the same controller so that it can be considered a global controller [7].

\section{SimUlation RESUlTS UNDER FAULTS}

In this section, the three different LQG design approaches will be tested under four kinds of faults described previously. Just as in the healthy situation, a step input will be used firstly, and then, a ramp signal will be created as the input to test the tracking ability of the systems.
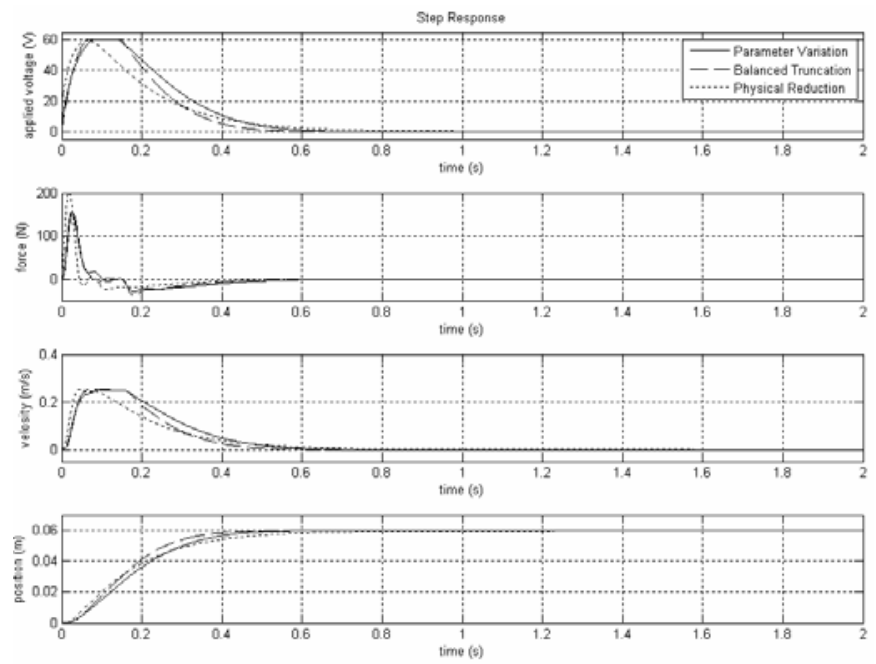

Fig. 8 Simulation result with bottom 1 actuator locked up

TABLE II

STEP RESPONSE PERFORMANCE OF SEREIS IN PARALLEL TWO BY TWO STRUCTURE IN TIME AND FREQUENCY DOMAIN

\begin{tabular}{|c|c|c|c|c|c|}
\hline & Situations & $\mathrm{FV}(\mathrm{m})$ & $\mathrm{RT}(\mathrm{s})$ & $\mathrm{ST}(\mathrm{s})$ & OS \\
\hline \multirow{3}{*}{ Healthy } & Parameter variation & 0.06 & 0.20 & 0.45 & $4.3 \%$ \\
\hline & Balanced truncation & 0.06 & 0.20 & 0.44 & $4.2 \%$ \\
\hline & Physical reduction & 0.06 & 0.23 & 0.36 & $0 \%$ \\
\hline \multirow{3}{*}{$\begin{array}{c}\text { Over } \\
\text { heating }\end{array}$} & Parameter variation & 0.06 & 0.17 & 0.51 & $9.5 \%$ \\
\hline & Balanced truncation & 0.06 & 0.17 & 0.52 & $9.8 \%$ \\
\hline & Physical reduction & 0.06 & 0.18 & 0.25 & $0 \%$ \\
\hline \multirow{3}{*}{$\begin{array}{l}\text { Open } \\
\text { circuit }\end{array}$} & Parameter variation & 0.06 & 0.20 & 0.45 & $4.3 \%$ \\
\hline & Balanced truncation & 0.06 & 0.20 & 0.44 & $4.0 \%$ \\
\hline & Physical reduction & 0.06 & 0.23 & 0.36 & $0 \%$ \\
\hline \multirow{3}{*}{ Backlash } & Parameter variation & 0.06 & 0.20 & 0.45 & $4.3 \%$ \\
\hline & Balanced truncation & 0.06 & 0.20 & 0.44 & $4.2 \%$ \\
\hline & Physical reduction & 0.06 & 0.23 & 0.36 & $0 \%$ \\
\hline \multirow{4}{*}{ Lockup } & Parameter variation & 0.06 & 0.31 & 0.43 & $0 \%$ \\
\hline & Balanced truncation & 0.06 & 0.31 & 0.43 & $0 \%$ \\
\hline & Physical reduction & 0.06 & 0.38 & 0.64 & $0 \%$ \\
\hline & & PM(deg) & \multicolumn{2}{|c|}{$\mathrm{GM}(\mathrm{dB})$} & $\mathrm{BW}(\mathrm{Hz})$ \\
\hline \multirow{3}{*}{ Healthy } & Parameter variation & 64.5 & \multicolumn{2}{|c|}{20.8} & 2.14 \\
\hline & Balanced truncation & 64.3 & \multicolumn{2}{|c|}{19.3} & 2.18 \\
\hline & Physical reduction & 76.2 & \multicolumn{2}{|c|}{20.7} & 1.72 \\
\hline \multirow{3}{*}{$\begin{array}{c}\text { Over } \\
\text { heating }\end{array}$} & Parameter variation & 57.8 & \multicolumn{2}{|c|}{17.8} & 2.7 \\
\hline & Balanced truncation & 57.4 & \multicolumn{2}{|c|}{16.1} & 2.75 \\
\hline & Physical reduction & 70.9 & \multicolumn{2}{|c|}{20.5} & 2.42 \\
\hline \multirow{3}{*}{$\begin{array}{l}\text { Open } \\
\text { circuit }\end{array}$} & Parameter variation & 63.9 & \multicolumn{2}{|c|}{16} & 2.14 \\
\hline & Balanced truncation & 63.8 & \multicolumn{2}{|c|}{15.3} & 2.24 \\
\hline & Physical reduction & 75.7 & \multicolumn{2}{|c|}{14.0} & 1.78 \\
\hline \multirow{3}{*}{ Backlash } & Parameter variation & 64.4 & \multicolumn{2}{|c|}{20.8} & 2.13 \\
\hline & Balanced truncation & 64.3 & \multicolumn{2}{|c|}{19.2} & 2.16 \\
\hline & Physical reduction & 76.1 & \multicolumn{2}{|c|}{20.6} & 1.61 \\
\hline \multirow{3}{*}{ Lockup } & Parameter variation & 72.1 & \multicolumn{2}{|c|}{26.4} & 1.70 \\
\hline & Balanced truncation & 72.2 & \multicolumn{2}{|c|}{24.3} & 1.32 \\
\hline & Physical reduction & 80.9 & \multicolumn{2}{|c|}{27.7} & 1.04 \\
\hline
\end{tabular}




\section{A. Step Response}

As in the healthy situation, a $0.06 \mathrm{~m}$ step is chosen as the input command. As an example, the simulation result with one sub-actuator locked up using three design approaches is shown in Fig 8. Bottom 1 as shown in Fig 4 is chosen as the faulty sub-actuator, and the applied voltage is limited at $60 \mathrm{~V}$. Note that, this will be the same in the simulations for other three types of fault.

Compared with the result for the healthy situation, which was shown in Fig 6, a decrease in performance can be found (as expected). More details about the performance in both time and frequency domain is given in the Table II, where FV is Final value of position, RT is Rise time, ST is Settling time, and OT is overshoot, GM is Gain margin, PM is Phase margin, $\mathrm{BW}$ is Bandwidth.

From the performance table, decreases in the performance in both time and frequency domain can be found under all four types of fault. It is also noted that the performance is similar for all three design (reduction) approaches.

\section{B. Ramp Response}
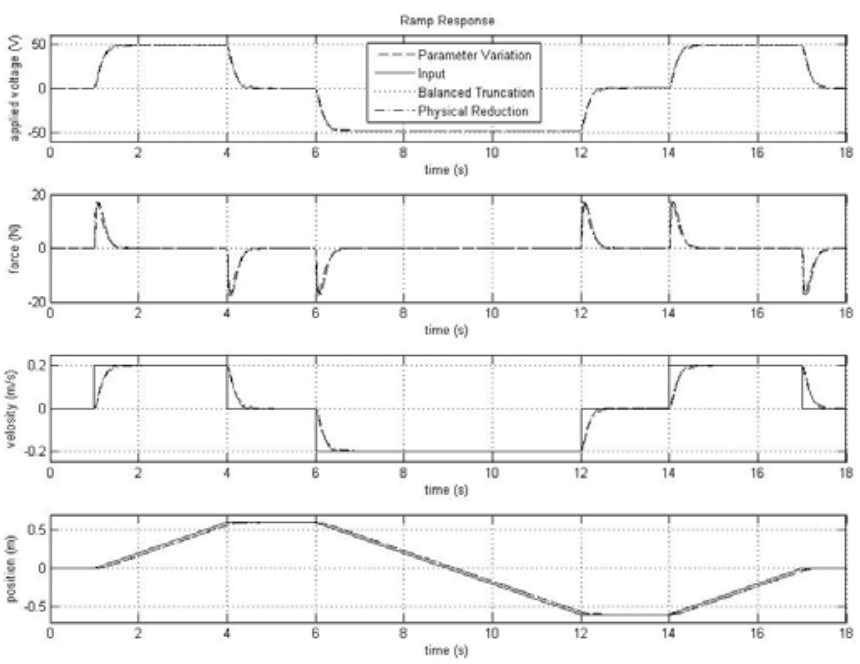

Fig. 9 Simulation result with bottom 1 actuator locked up

TABLE III

RAMP RESPONSE PERFORMANCE OF SEREIS IN PARALLEL TWO BY TWO STRUCTURE

\begin{tabular}{cccc}
\hline Situations & $\begin{array}{c}\text { Coefficient of } \\
\text { determination for } \\
\text { position }\end{array}$ & $\begin{array}{c}\text { Coefficient of } \\
\text { determination for } \\
\text { velocity }\end{array}$ \\
\hline \multirow{2}{*}{ Healthy } & Parameter variation & $99.79 \%$ & $96.69 \%$ \\
& Balanced truncation & $99.80 \%$ & $95.72 \%$ \\
\hline \multirow{2}{*}{ Over } & Physical reduction & $99.76 \%$ & $95.17 \%$ \\
\hline \multirow{2}{*}{ Opeating } & Parameter variation & $99.83 \%$ & $96.85 \%$ \\
& Balanced truncation & $99.84 \%$ & $95.79 \%$ \\
& Physical reduction & $99.81 \%$ & $95.46 \%$ \\
\hline \multirow{2}{*}{ Backlash } & Parameter variation & $99.79 \%$ & $96.62 \%$ \\
& Balanced truncation & $99.80 \%$ & $95.64 \%$ \\
& Physical reduction & $99.76 \%$ & $95.11 \%$ \\
\hline \multirow{2}{*}{ Lockup } & Parameter variation & $99.79 \%$ & $96.57 \%$ \\
& Balanced truncation & $99.80 \%$ & $95.63 \%$ \\
& Physical reduction & $99.76 \%$ & $95.07 \%$ \\
\hline & Parameter variation & $99.55 \%$ & $94.36 \%$ \\
& Balanced truncation & $99.56 \%$ & $94.43 \%$ \\
\hline
\end{tabular}

In this paper, a novel type of high redundancy actuator with the capability of fault tolerance is introduced. An individual electro-mechanical actuator and a two by two configuration HRA have been modeled. A LQG controller has been presented for the individual actuator, followed by three different LQG designs for the HRA (series-in-parallel two by two configuration). These were tested under typical fault conditions. The results show that the high redundancy actuator does have a fault tolerance capability without the need to reconfigure. Based in the performance alone there was little difference between the three LQG design approaches. But when complexity of the design process and the controller order are taken into account, the LQG design using a physically motivated reduced order model is judged to be the best approach. Other on-going work is investigating the design of alternative actuator system structures. Future studies will extend the concepts to 3-by-3 structures and 
higher, and will evaluate other controller designs based on modern robust control methods, i.e. $H_{2}$ and $H_{\infty}$. Other structures of controller, including inner loops or local controllers $[5,6]$, will also be considered. It is also intended to develop a lab scale actuator to demonstrate the concept in hardware. By doing so the authors hope to gain an insight into the practicality and rough cost implications of such a system.

\section{Acknowledgement}

This project is a cooperation of the Control Systems group at Loughborough University, the Systems Engineering and Innovation Centre (SEIC), and the actuator supply SMAC UK limited. The work is supported by funding from the UK's research council EPSRC (reference EP/D078350) and via a department research scholarship.

\section{REFERENCES}

[1] R. Patton, "Fault Tolerant Control: The 1997 Situation," SAFE-PROCESS'97, IFAC Symposium on fault detection, supervision and safety, Kingston Upon Hull, U.K.

[2] M. Blanke, M. Kinnaert, J. Lunze, and M. Staroswiecki, Diagnosis and Fault-Tolerant Control, Second Edition, Springer 2006

[3] D. Briere, C. Favre, and P. Traverse, "A family of Fault Tolerant Systems: Electrical flight controls from Airbus A320/A330/A340 to future military transport aircraft," Microprocessors and Microsystems, Volume 19, Number 2, pp. 75-82

[4] T. Ford, "Actuation systems development," Aircraft Engineering and Aerospace Technology, Volume 70, Number 4, pp. 265-270

[5] T. Steffen, J. Davies, R. Dixon, and R.M. Goodall, "Using a Series of Moving Coils as a High Redundancy Actuator," in Preprint of the IFAC Conference for Advanced Intelligent Mechatronics (AIM), Zurich, 2007.

[6] X. Du, R. Dixon, R.M. Goodall, and A.C. Zolota, "Assessment Of Strategies For Control Of High Redundancy Actuators," ACTUATOR 2006, Germany

[7] X. Du, R. Dixon, R.M. Goodall, and A.C. Zolota, "Modelling And Control Of A Highly Redundant Actuator," CONTROL 2006, Scotland

[8] J. H. Baek, Y.K. Kwak, and S. H. Kim, "On the frequency bandwidth change of a servo system with a gear reducer due to backlash and motor input voltage," Archive of Applied Mechanics, Volume 73, pp.367-376

[9] G. Obinata, and B. D.O. Anderson, Model Reduction for Control System Design, Springer 2001 